05

\title{
Анализ распределения магнитного поля и оценка критической плотности тока на основе данных магнитооптических исследований сверхпроводящих ленточных композитов
}

\author{
(С) А.Э. Применко, ${ }^{1}$ М.А. Осипов, ${ }^{2}$ И.А. Руднев ${ }^{2}$ \\ ${ }^{1}$ Московский государственный технический университет им. Н.Э.Баумана, \\ 105005 Москва, Россия \\ ${ }^{2}$ Национальный исследовательский ядерный университет „МИФИ“, \\ 115409 Москва, Россия \\ e-mail: iarudnev@mephi.ru
}

(Поступило в Редакцию 3 октября 2016 г. В окончательной редакции 7 февраля 2017 г.)

Представлен подробный анализ результатов проникновения магнитного потока в высокотемпературные сверхпроводящие композиты второго поколения. Экспериментальные наблюдения распределения магнитного потока были проведены с помощью техники магнитооптической визуализации при различных температурах от 4.2 до $80 \mathrm{~K}$. Показано совпадение экспериментальных данных с результатами аналитических и получисленных расчетов профилей магнитного поля, выполненного для режимов полного и неполного проникновения магнитного поля в сверхпроводящие композиты. Рассмотрена методика оценки критической плотности тока из измерений топографии поперечного магнитного потока, а также предложен метод оценки критической плотности тока лент из измерений касательной компоненты поля.

DOI: 10.21883/JTF.2017.09.44907.2054

\section{Введение}

Тонкие сверхпроводящие пленки в критическом состоянии являются интересными объектами для исследований как с фундаментальной, так и прикладной точки зрения. Значительную актуальность таким исследованиям придает бурное развитие технологий создания ленточных ВТСП композитов второго поколения (другое название СС-ленты, от английского „coated conductor“), в которых ключевым токонесущим элементом является тонкий слой высокотемпературного сверхпроводника. Такие ленты имеют высокие транспортные характеристики. Так, в нулевом внешнем магнитном поле при температуре $77 \mathrm{~K}$ плотность критического тока $J_{c}$ превышает $10^{6} \mathrm{~A} / \mathrm{cm}^{2}$, а при температуре $4.2 \mathrm{~K}$ величина $J_{c} \sim 10^{6} \mathrm{~A} / \mathrm{cm}^{2}$ достигается в магнитных полях порядка нескольких десятков Тесла.

ВТСП ленты представляют собой структуру со сложной архитектурой слоев, нанесенных на гибкую металлическую подложку. Непосредственно на металлическую подложку нанесены буферные слои, на них осажден слой ВТСП толщиной $\sim 1 \mu \mathrm{m}$. Сверху слой ВТСП закрыт защитными покрытиями из серебра и меди. Из-за наличия высокопроводящего защитного покрытия для исследования токонесущих свойств лент должны использоваться бесконтактные методы. Одним из таких методов могло бы быть измерение кривых намагничивания и определение критического тока по ширине петли гистерезиса. Но данная методика требует помещения всего образца в область между измерительными катушками, что невозможно для длинных лент. Другой альтернативой являются методы, основанные на измерении распределения магнитного поля вне ленты и последующем обсчете для оценки величины критических токов. При этом распределение магнитного поля измеряется с помощью линейки преобразователей Холла, а измерения проводятся для длинной ленты при непрерывной перемотке с бобины на бобины [1-3]. Получение распределения магнитного поля в лентах с высоким пространственным разрешением может быть достигнуто использованием различных методик. Наиболее эффективными методами такого типа являются Холловская сканируюшая микроскопия [4,5] и магнитооптическая визуализация (МОВ) [6,7]. К настоящему времени проведен ряд исследований СС-лент с применением техники магнитооптической визуализации. Так, в $[8,9]$ исследовалось распределение плотности постоянного и переменного транспортного тока, в работе [10] распространение нормальной зоны при возникновении тепловой флуктуации. МОВ методика использовалась для изучения контроля качества ВТСП лент, спаев и кабелей на основе ВТСП лент [11-16]. Также МОВ применялась для исследований механических свойств СС-лент $[17,18]$. В работах $[19,20]$ представлены данные магнитооптических (MO) экспериментов по намагничиванию и перемагничиванию ВТСП ленты при различных температурах. Было продемонстрировано, что перемагничивание СС-ленты сопровождается возникновением и распространением волны аннигиляции магнитного потока. Свойства волны аннигиляции магнитного потока были предсказаны ранее как результат численного анализа вихревой системы с дефектами методом Монте-Карло [21]. 
Вместе с тем в литературе практически отсутствует подробный анализ проникновения магнитного потока в ВТСП ленты в режиме формирования критического состояния. В цитированных выше работах также не рассматривается определение критического тока из профилей магнитного поля. Целью настоящей работы является детальный анализ результатов магнитооптических измерений топографии поля в ВТСП композитных лентах при различных температурах с целью исследования распределения поля и разработки простого метода оценки критической плотности тока из этих измерений. Сложность интерпретации данных заключается в том, что распределение магнитного поля в тонкой пленке, помещенной в поперечное магнитное поле, заметно отличается от картины предсказываемой простой моделью Бина [22]. В отличие от массивного образца в тонкой пленке в мейсснеровской области имеются ненулевые плотности тока, а экранирование магнитного поля осуществляется только для поперечной компоненты [23,24]. Для интерпретации результатов МО экспериментов нами были получены (в рамках модели $J_{c}=$ const) аналитические соотношения для распределения магнитного поля. Учтена конечность толщины пленки (в отличие от имеющихся в литературе подходов) и получено распределение для обеих компонент магнитного поля внутри и вне сверхпроводника. Для полей, больших поля полного проникновения (в том числе для участка перемагничивания), получены аналитические выражения. Для начального участка кривой намагничивания получено получисленное решение. В настоящей работе будет показано, что расчетные профили поля удовлетворительно согласуются с экспериментальными данными. Для оценки критической плотности тока из измерений топографии поперечного поля будет предложена методика, малочувствительная к точной форме токовых линий. Кроме того, мы покажем, что наблюдаемая в эксперименте асимметрия профиля поля связана, вероятно, с небольшим отклонением от параллельности МО пленки и плоскости СП ленты, что возможно из-за наличия небольшой поперечной кривизны реальных ВТСП лент на металлических подложках. Указана перспективность оценки критической плотности тока лент из измерений касательной компоненты поля, и приведена возможная принципиальная схема эксперимента.

\section{1. Экспериментальные методы}

Метод магнитооптической визуализации основан на использовании эффекта Фарадея: при распространении в веществе линейно поляризованного света вдоль силовых линий магнитного поля происходит поворот плоскости поляризации проходящего излучения. Угол поворота $\varphi$ пропорционален длине пути света в веществе L, напряженности магнитного поля $\mathrm{H}$ и постоянной Верде $\mathrm{C}_{\mathrm{V}}$ : $\varphi=\mathrm{C}_{\mathrm{V}} \mathrm{HL}$. В качестве активной среды применяется магнитная индикаторная пленка, которая представляет собой слой магнитного вещества (иттрий-железистый гранат, легированный висмутом), который с одной стороны покрыт алюминием для создания зеркальной отражающей поверхности. Индикаторная пленка свободно помещается на сверхпроводящую ленту. После охлаждения сверхпроводника ниже критической температуры включают внешнее магнитное поле, которое проникает в сверхпроводник. При этом в силу относительно малых толщин магнитооптической индикаторной пленки планарное распределение магнитной индукции в МО пленке соответствует локальному распределению магнитной индукции в сверхпроводнике. В поляризованном свете области с большим магнитным полем имеют более яркое изображение. Из матрицы интенсивности отраженного света с помощью калибровочной кривой получают двумерную матрицу локальной магнитной индукции. Применяемая нами методика получения и обработки МО изображений аналогична описанной в литературе [6,7]. В качестве объектов исследования мы использовали коммерческие ВТСП ленты компании SuperPower с критической температурой 89-90 К и величиной критического тока при $T=77 \mathrm{~K} 100-120 \mathrm{~A}$ при ширине ленты $4 \mathrm{~mm}$.

\section{2. Экспериментальные результаты}

Типичное магнитооптическое изображение проникновения магнитного потока в ВТСП ленту показано на рис. 1. Более светлые области соответствуют более высокому значению поля. При повышении внешнего магнитного поля фронт проникновения магнитного потока продвигается вглубь образца (рис. 2). Изменение температуры приводит к изменению глубины проникновения фронта магнитного потока (рис. 3). После снятия

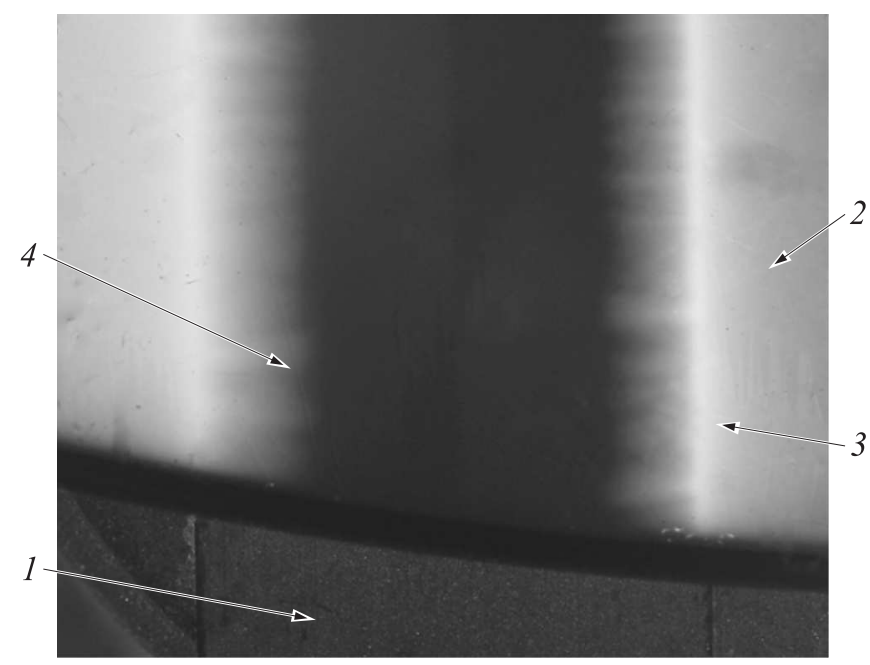

Рис. 1. Типичное магнитооптическое изображение ВТСП ленты, находящейся во внешнем поперечном магнитном поле $28 \mathrm{mT}$. Температура $60 \mathrm{~K}: 1-$ ВТСП лента, 2 - область внешнего магнитного поля, 3 - область намагничивания, 4 передний фронт проникающего в ленту магнитного потока. 

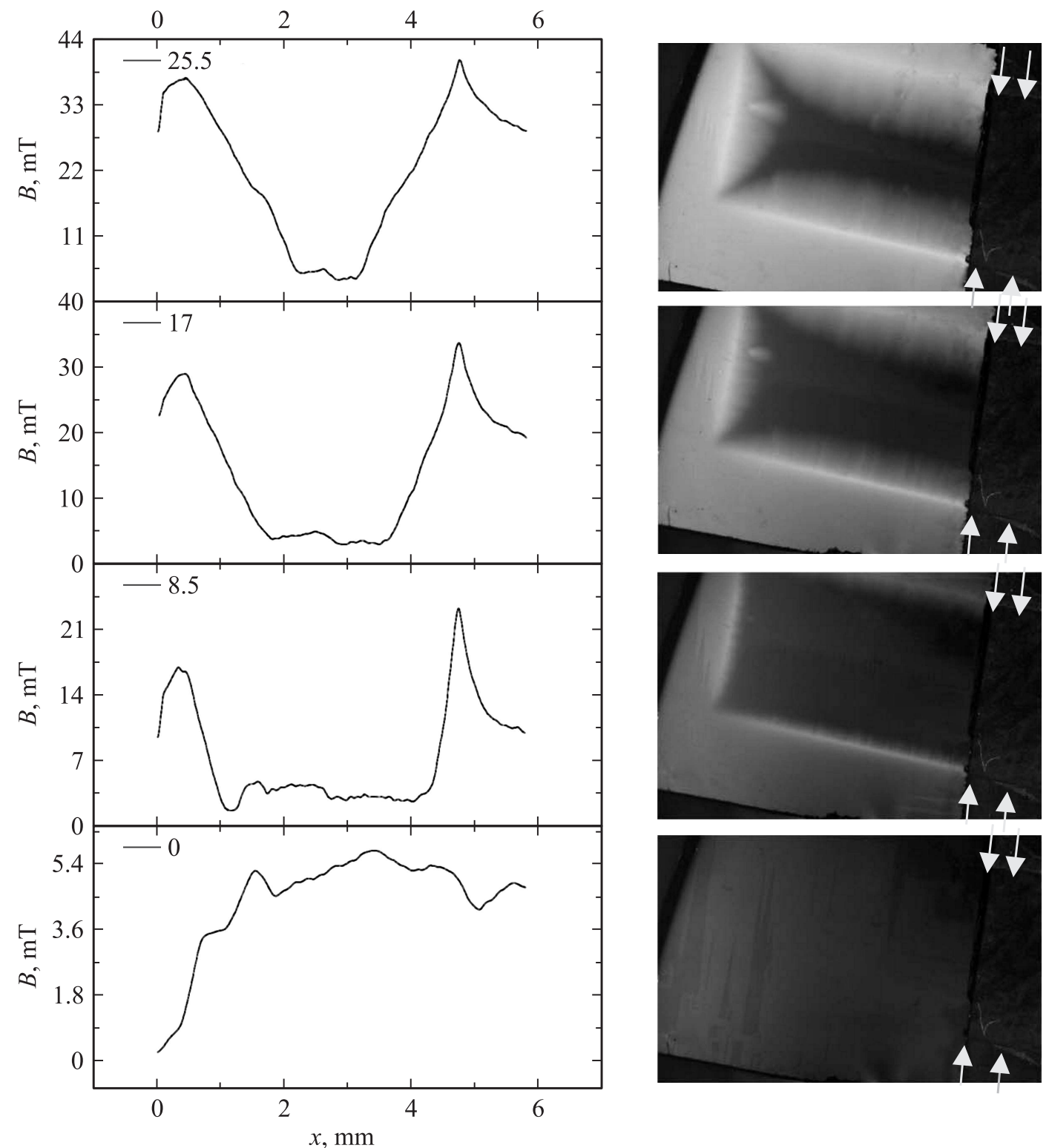

Рис. 2. Магнитооптические изображения проникновения магнитного потока при увеличении внешнего магнитного поля при $T=77 \mathrm{~K}$. Величина магнитного поля приведена в левом верхнем углу графика с профилем поля. Справа от индикаторной пленки видны края ВТСП ленты в медной оболочке (отмечены стрелками).

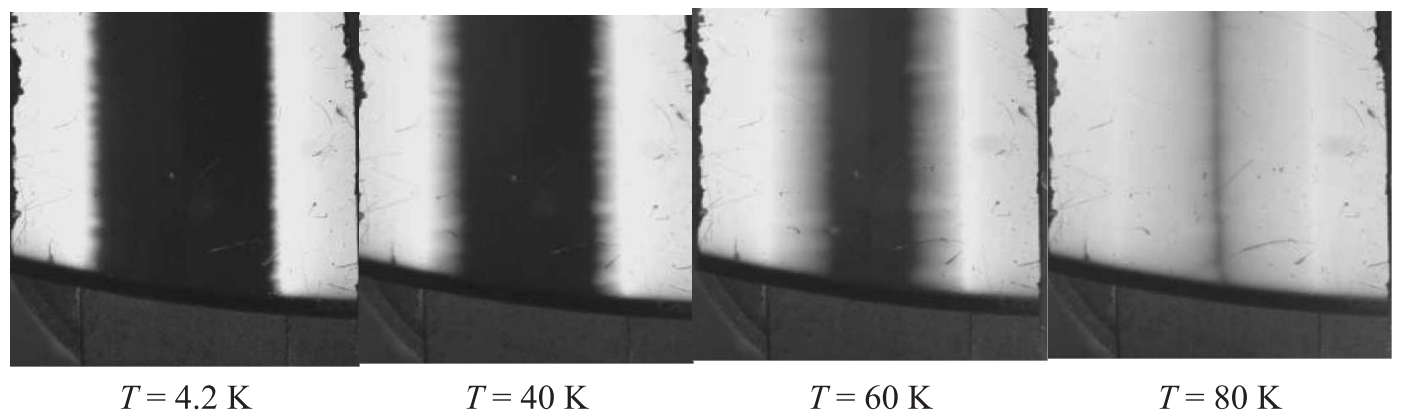

Рис. 3. МО изображения распределения перпендикулярной составляющей магнитного поля над СП лентой при различных значениях температуры. 


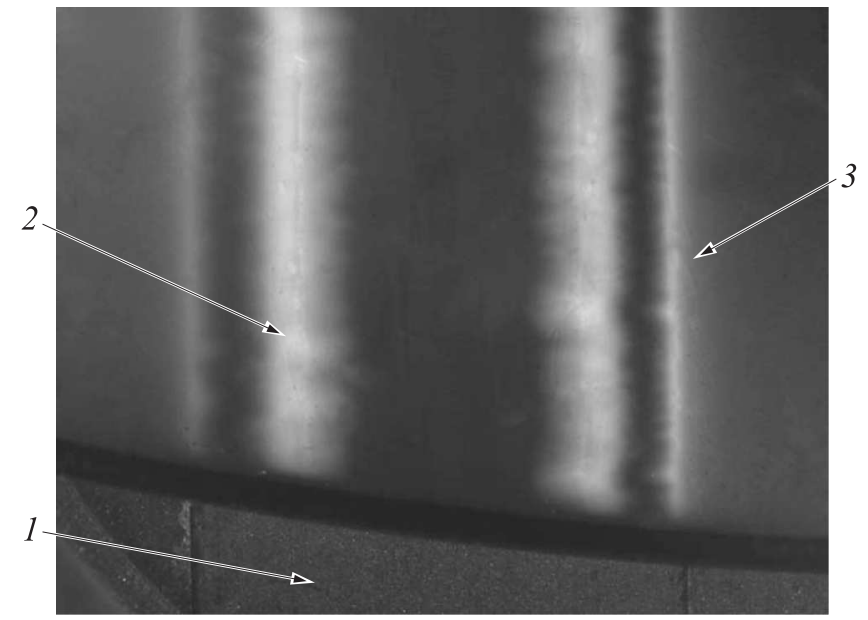

Рис. 4. Магнитооптическое изображение остаточной намагниченности ВТСП ленты, после приложения и снятия внешнего поля $42.3 \mathrm{mT}$. Температура $60 \mathrm{~K}: 1$ - ВТСП лента, 2 - область магнитного поля обратного знака, 3 - область захваченного магнитного потока.

внешнего поля в сверхпроводнике остается захваченный магнитный поток, положение и величина которого зависят от значения локального критического тока. Этот захваченный магнитный поток (при нулевом внешнем поле) формируется за счет закрепления вихрей Абрикосова на центрах пиннинга (природных и технологических дефектах, неоднородностях, вторых фазах и т.п.). При изменении знака внешнего поля в пленку с краев начинают проникать вихри обратного знака (антивихри), что приводит к движению фронта магнитной индукции противоположного знака. Антивихри и запиннингованные вихри аннигилируют, что приводит к появлению отчетливой области с $B=0$ - волной аннигиляции магнитного потока (рис. 4).

\section{3. Анализ экспериментальных результатов}

\section{1. Вычисление распределения магнитного поля в сверхпроводящей ленте в критическом состоянии для различных участков кривой намагничивания}

Распределение магнитного поля, создаваемого сверхпроводящей пленкой, было вычислено в приближении бесконечной полосы (шириной $w$ и толщиной $d$ ), постоянной критической плотности тока $J_{c}=$ const и пренебрежимо малым первым критическим полем $H_{c 1} \sim 0$.

Полное магнитное поле $B\left(r_{0}\right)$, непосредственно измеряемое в эксперименте

$$
B\left(r_{0}\right)=B_{a}+B^{\prime}\left(r_{0}\right),
$$

где $B_{a}-$ внешнее приложенное поле, $B^{\prime}\left(r_{0}\right)-$ поле, создаваемое лентой (собственное поле ленты).
Для данной геометрии собственное поле рассчитывается путем интегрирования закона Био-Савара по поперечному сечению ленты $S_{0}$

$$
B^{\prime}\left(r_{0}\right)=\int_{S_{0}} \frac{\mu_{0}}{2 \pi} \frac{J(r)\left(r_{0}-r\right)}{\left|r_{0}-r\right|^{2}} d s(r) .
$$

Здесь $B^{\prime}\left(r_{0}\right)$ - собственное магнитное поле в точке $r_{0}$, $J(r)$ - плотность тока в точке $r$. Распределение плотности тока $J(r)$ зависит от предыстории намагничивания ленты. Ниже мы рассмотрим основные участки кривой намагничивания и соответствующие им распределения плотности тока, использованные в наших расчетах.

1. На участке начального намагничивания в сверхпроводнике сосуществуют мейсснеровская область и область критического состояния. Результирующее поле складывается из поля от токов, текущих в мейсснеровской области, поля от токов, текущих в области критического состояния, и внешнего поля. В отличие от продольной геометрии в мейсснеровской области плотность тока $J$ не равна нулю, хотя нормальная компонента поля обращается в нуль. При расчетах мы считаем плотность тока в мейсснеровской области независящей от координаты $z$ и меняющейся с координатой $x$ согласно соотношению из работы [24]:

$$
\begin{gathered}
j(x)=\frac{2}{\pi} J_{c} \operatorname{arctg}\left(\frac{\sqrt{1-\left(\frac{x_{p}}{w / 2}\right)^{2}} x}{\sqrt{x_{p}^{2}-x^{2}}}\right), \quad|x|<X_{p}, \\
j(x)=J_{c}, \quad x \geq X_{p} .
\end{gathered}
$$

Здесь $X_{p}$ - положение границы мейсснеровской области, $J_{c}-$ критическая плотность тока, $w-$ ширина ленты.

Магнитное поле, создаваемое токами текущими в области критического состояния (где $J=J_{c}$ ), может быть найдено аналитически как поле от двух бесконечных полосок ширины $w$ и толщины $d$, средние линии которых расположены на расстоянии $X_{p}+w / 2$. Формулы для поля полоски приведена в Приложении А.

Магнитное поле, создаваемое токами, текущими в мейсснеровской области (где $J=j(x)$ ), вычислим по следующему получисленному алгоритму. Разобьем мейсснеровскую область на вертикальные полоски (рис. 5,a) (с координатами центров полос $x_{i}$ ), плотность тока $J_{i}=j\left(x_{i}\right)$. Магнитное поле полоски задается аналитически (см. Приложение А). Оно не имеет особенностей ни в одной точке пространства. Суммируя вклады от всех полосок, вычисляем магнитное поле, создаваемое токами, текущими в мейсснеровской области. Использование такого метода позволяет получить гладкое решение при сравнительно небольшом числе точек разбиения.

Положение границы между мейсснеровской областью и областью критического состояния определяется условием, что в мейсснеровской области поле $B_{z}$ должно 


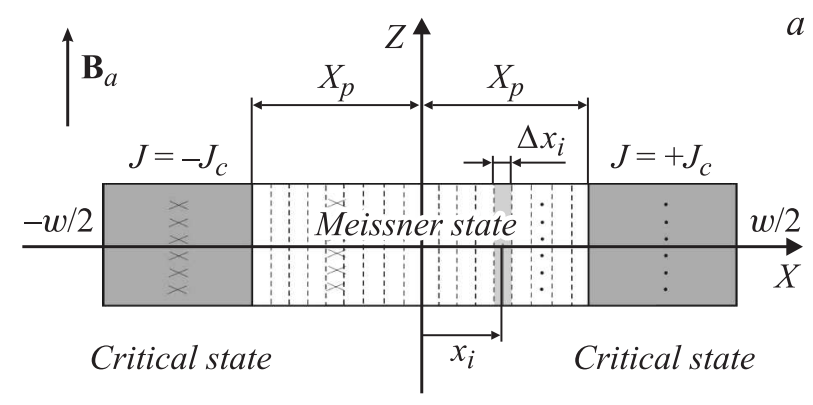

$a$
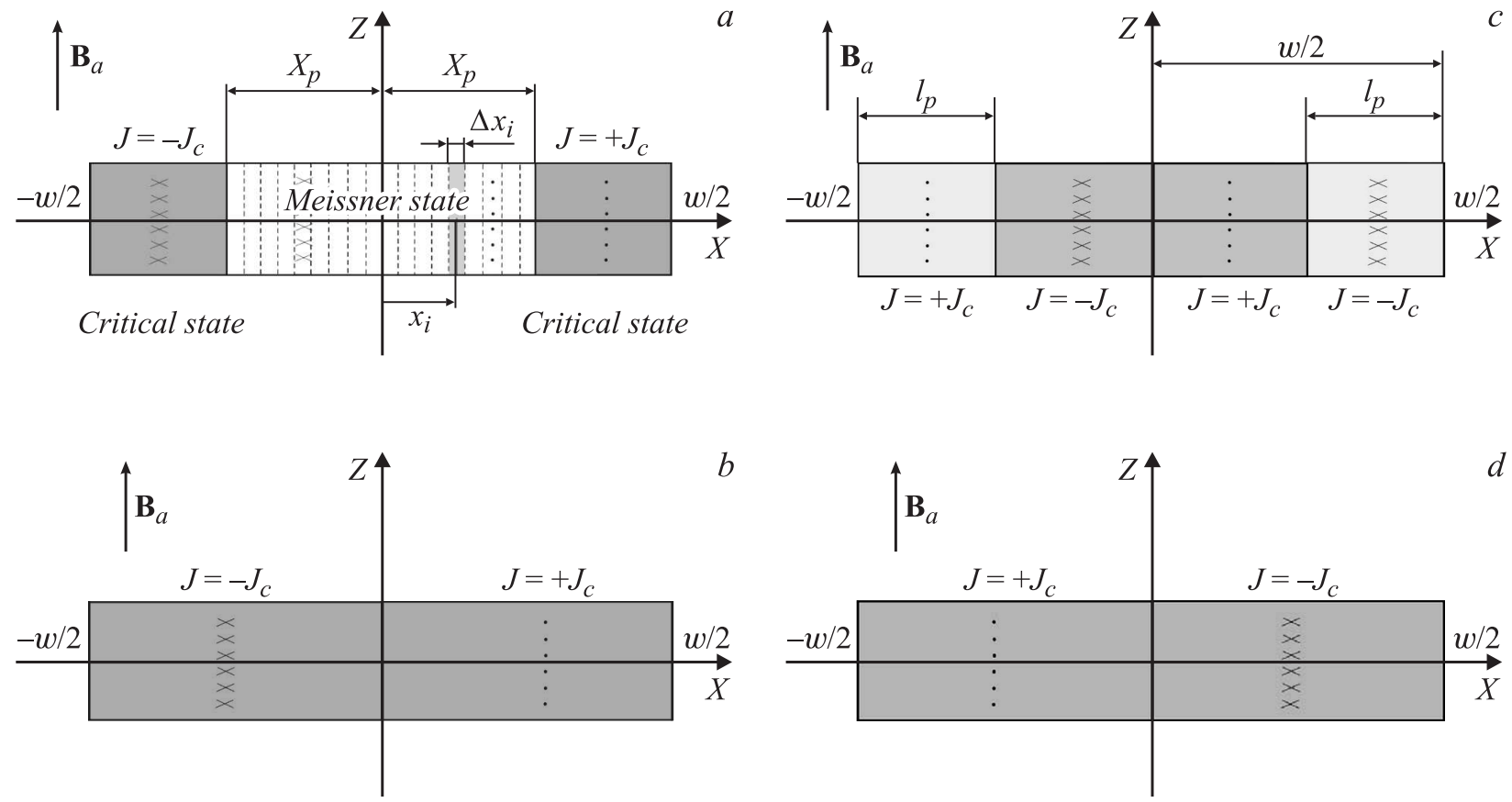

$b$

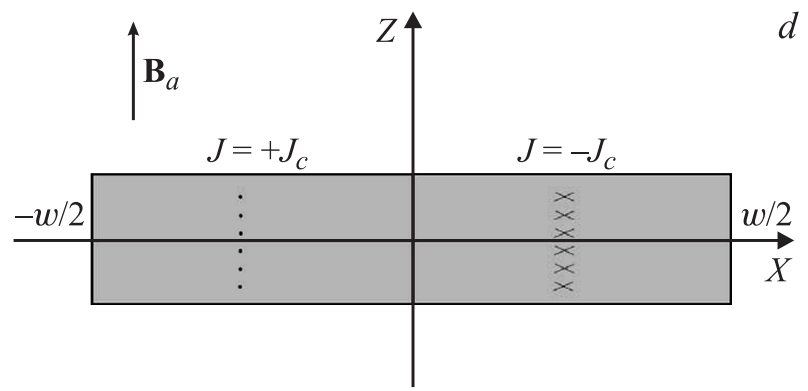

Рис. 5. Распределение токов в СП ленте на различных участках кривой намагничивания: $a-$ участок начального ввода, $b-$ участок насыщения после начального ввода, $c-$ участок перемагничивания, $d-$ участок насыщения после перемагничивания.

равняться нулю:

$$
B_{z}(x, z)=B_{a}+B_{z}^{\prime}(x, z)=0 .
$$

Рассчитав для заданного положения границы мейсснеровской области собственное поле $B_{z}$ в центре образца, можно найти внешнее магнитное поле, соответствующее данному положению границы мейсснеровской области.

2. На участке насыщения (после участка начального ввода) весь образец находится в критическом состоянии, плотность тока $|\mathbf{J}|=J_{c}$, и поле может быть вычислено аналитически. Собственное магнитное поле создается двумя полосками (рис. $5, b)$ толщиной $d$ и шириной $w / 2$ несущими ток плотности $J_{c}$. Центр первой полоски расположен в точке $(x=w / 4, z=0)$, а центр второй в точке $(x=-w / 4, z=0)$ :

$$
B^{\prime}(x, z)=B_{+}(x, z)+B_{-}(x, z) .
$$

Здесь $B_{+}(x, z)$ - поле создаваемое первой полоской, $B_{-}(x, z)$ - поле, создаваемое второй полоской. В явном виде соотношение для магнитного поля $B^{\prime}(x, z)$ приведено в Приложении Б.

3. На участке перемагничивания в периферийной части сверхпроводника токи меняют свое направление на противоположное (рис. 5,c). В центральной части образца направление токов остается прежним.

Для расчета магнитного поля воспользуемся приближением, используемым в модели Бина для длинного цилиндра (или пластины) в продольном поле. В этой модели поле в центре образца остается неизменным в течение всего процесса перемагничивания. Величина этого поля зависит от максимального приложенного поля $B_{\max }$ и в нашем случае находится по формуле

$$
B_{z}(0,0)=B_{\max }+B_{z}^{\prime}(0,0) .
$$

Здесь $B_{z}(0,0)$ вычисляется по формуле (ПБ1) Приложения Б.

На участке перемагничивания собственное магнитное поле создается четырьмя полосками толщиной $d$ несущими ток плотности $J_{c}$ (рис. $\left.5, c\right)$.

$$
B^{\prime}(x, z)=\sum_{i=1}^{4} B_{i}(x, z),
$$

где $B_{i}(x, z)$ - поле, создаваемое первой $i$-ой полоской. Положение центров полосок и их ширины указаны на рис. $5, c$.

Из условия постоянства величины $B_{z}(0,0)$ в центре образца может быть найдено положение фронта перемагничивания $l_{p}$ и, следовательно, рассчитано распределение магнитного поля. Подробности такого расчета приведены в Приложении В.

4. На участке после перемагничивания по всему образцу текут токи плотности $J_{c}$ (в направлении, противоположном случаю 2 (рис. 5,d), и поле может быть вычислено, так же как и для участка 2 аналитически (Приложение Б).

\section{2. Применение модели к анализу результатов МО измерений}

Так как в расчетной модели принята однородная плотность критического тока, оценка величины $J_{c}$ яв- 


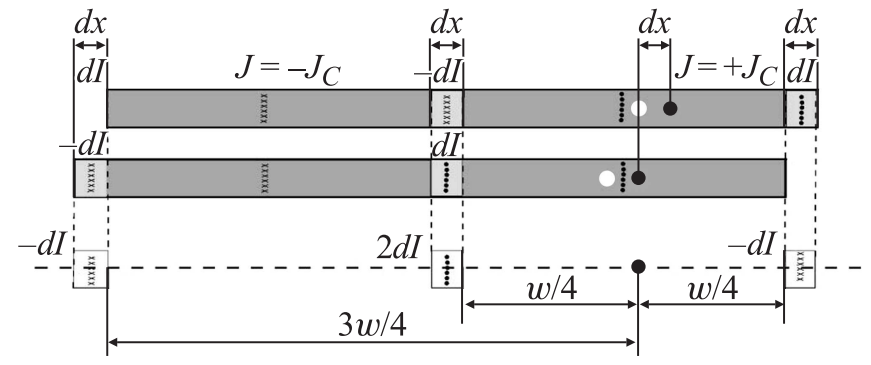

Рис. 6. Расчет наклона профиля поля в точке перегиба.

ляется ключевым моментом в анализе экспериментальных данных. Получив величину $J_{c}$, дальше возможно подставить ее в формулы, описывающие распределение поля в пространстве, и сравнить результаты расчета с экспериментом. В работе [25] нами было предложено оценивать величину критической плотности тока по наклону зависимости $B_{z}(x, z)$ в точке перегиба. Ниже мы приведем простое рассуждение, приводящее к формуле для такой оценки.

Для отдельной полоски с током центр перегиба находится в центре полосы. При наличии двух тонких $(a=w / d \gg 1)$ полосок с противоположными направлениями токов (как в состоянии насыщения, участок 2) можно считать, что точки перегиба находятся приблизительно в центре каждой из полосок. Найдем приращение поля при бесконечно малом смещении точки наблюдения из центра полоски на величину $d x$. Это приращение складывается из вкладов от бесконечно тонких токовых нитей, изображенных на рис. 6 темным цветом. Вклады от остальных частей полосок остаются неизменными.

Суммируя указанные вклады и деля результат на $d x$, получаем искомую формулу для наклона профиля поля в точке перегиба.

$$
\left(\frac{\partial B_{z}}{d x}\right)_{x=w / 4} \approx \mu_{0} J_{c} \frac{16}{3 \pi a} .
$$

Расчетная кривая $B_{z}(x, z)$ по формулам Приложения Б со значением $J_{c} \approx 1.1 \cdot 10^{6} \mathrm{~A} / \mathrm{cm}^{2}$ (полученным вышеописанным способом) и с $z / d=40$ показана на рис. 7. Наименьшая величина $B_{z} / \partial x$ хорошо определяется и слабо зависит от нарушений однородности плотности тока и расстояния $z$ от поверхности СП до МО пленки. Поэтому получаем простой и удобный способ оценки $J_{c}$ из измерений топографии поля. Отметим, что данный способ оценки критического тока корректнее, чем определение по разности полей в центре и на краю образца (поля в центре и на краю сильно зависят от отклонений реального распределения плотности тока от картины, принятой в модели). Если в центре или на краю образца имеется локальное ослабление критической плотности тока, то это приведет к заметному уменьшению пика в зависимости $B_{z}(x)$. Кроме того, любая экспериментальная методика сглаживает острые экстремумы в силу ограниченного пространственного разрешения.

Полученное выше значение критической плотности тока можно теперь использовать для вычисления профиля поля на других участках кривой намагничивания. При этом следует обратить внимание на следующие важные моменты.

Для лучшей подгонки расчетной кривой под экспериментальные данные необходимо учесть, что МО пленка отделена от сверхпроводящего слоя некоторым промежутком. Наиболее удовлетворительное соответствие между расчетом и экспериментом достигается при промежутке, равном 40 толщинам сверхпроводящей пленки (рис. 7).

Асимметрию реального профиля поля можно связать с непараллельностью МО пленки и сверхпроводящего слоя, возникающей вследствие неидеального прилегания МО пленки и сверхпроводящей ленты. В наших измерениях МО пленка свободно лежит на сверхпроводящей ленте.

Вследствие этого возможен небольшой угол $\theta$ между пленкой и лентой. Поскольку отношение $w / d$ очень велико, даже малый угол $\theta$ приводит к сильному удалению части МО пленки от поверхности сверхпроводника в область пространства с меньшим полем. Влияние вклада $x$-компоненты поля на асимметрию профиля в нашем случае несущественно, так как угол $\theta$ порядка $1^{\circ} \mathrm{C}$. В качестве примера на рис. 8 приведено сопоставление расчетной кривой (значение критической плотности тока получено выше) и экспериментальных данных для случая частичного проникновения потока. При расчетах принято, что угол между МО пленкой и СП составляет $1.5^{\circ}$.

Хотя в МО эксперименте измеряется только поперечная составляющая магнитного поля, интересно также

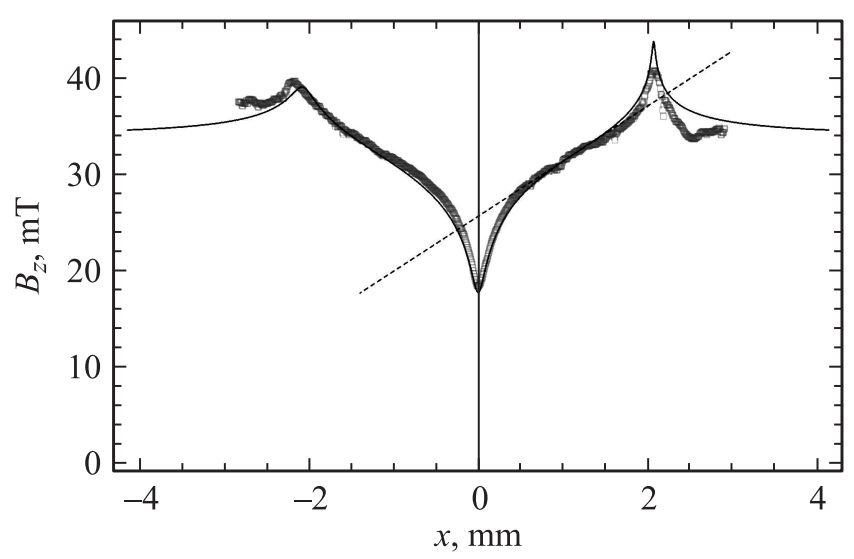

Рис. 7. Профиль поля в „насыщении“ после начального ввода, внешнее поле $42.3 \mathrm{mT}$. Расчетная кривая получена по формулам (ПБ1) Приложения Б с учетом непараллельности ленты и $\mathrm{MO}$ пленки (угол $\theta=1.5^{\circ}$ ) для $z / d=40$. Значение $J_{c} \approx 1.1 \cdot 10^{6} \mathrm{~A} / \mathrm{cm}^{2}$ определено по наклону $\partial B_{z} / \partial x$ в точке перегиба (формула (8)). 


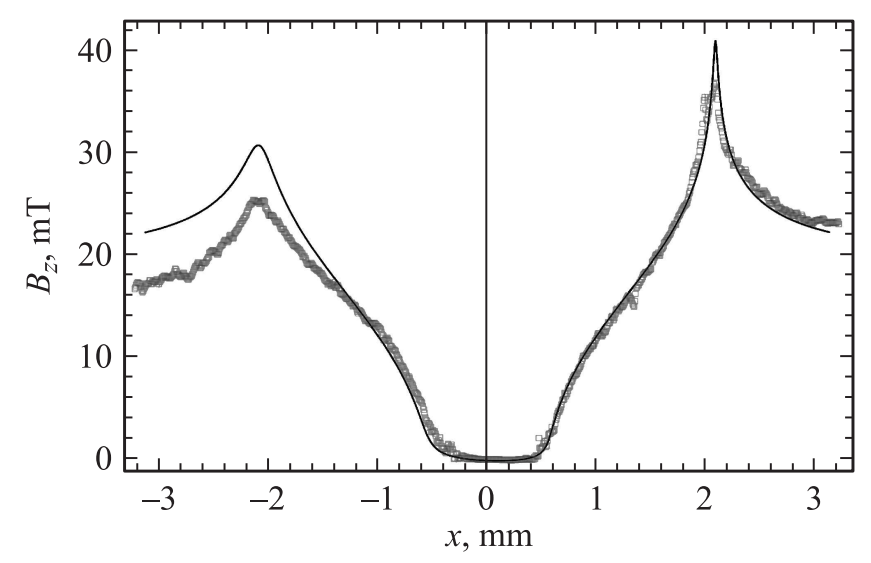

Рис. 8. Сопоставление экспериментальных данных и расчетной кривой для поперечной компоненты поля. Внешнее поле $B_{a}=18.8 \mathrm{mT}$, угол между МО пленкой и СП $\theta=1.5^{\circ}$. Значение критической плотности тока $J_{c}$ рассчитано по данным на рис. 7.

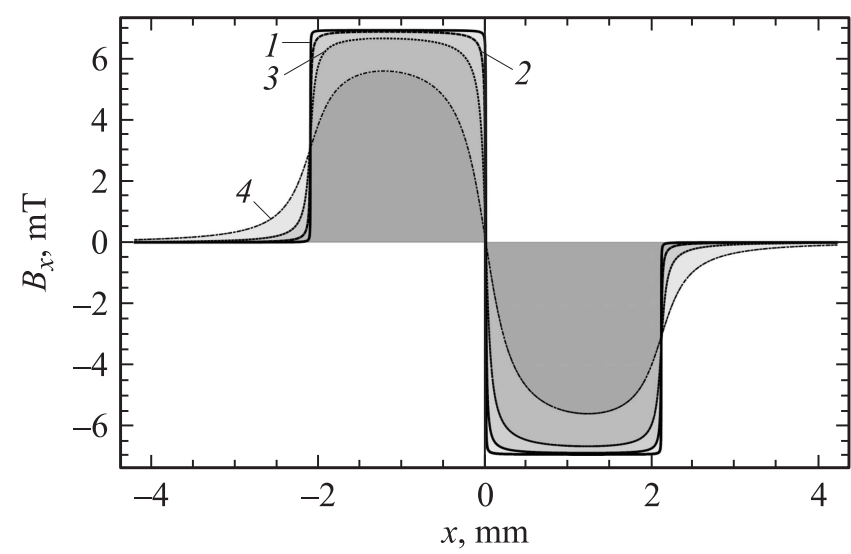

Рис. 9. Распределение касательной компоненты $B_{x}$ магнитного поля для различных расстояний от МО пленки до СП. Кривая $1-z / d=1$, кривая $2-10$, кривая $3-50$, кривая $4-250$. Значение $J_{c}$ для расчета взято таким же, как и на предыдущих рисунках.

проанализировать поведение касательной компоненты в различных режимах. Так, в режиме начального проникновения потока в мейсснеровской области касательная компонента обращается в нуль только в нулевой плоскости. Для координаты $z$, отличной от нуля, в мейсснеровской области имеем $B_{x}$, отличную от нуля всюду, кроме начала координат. Такое распределение представляется вполне разумным, так как тонкая пленка (толщиной порядка глубины проникновения $\lambda$ ) не может экранировать поле, параллельное ее поверхности.

В области насыщения распределение для касательной компоненты особенно простое. Внутри образца оно линейно зависит от координаты $z$, достигая максимального значения на поверхности ленты. Профиль поля на поверхности по форме практически совпадает с профилем тока (рис. 9). Это еще более ярко выражено на участке перемагничивания, где точки резкого излома профиля поля соответствуют перемене направления критических токов.

По мере удаления от поверхности сверхпроводника профиль касательной компоненты все больше сглаживается, но даже на расстояниях 50 толщин отличие от значения на поверхности составляет все еще 0.97 от значения на поверхности (рис. 9).

Простая связь между касательной компонентой магнитного поля на поверхности ленты и плотностью тока в ней делают перспективным разработку метода оценки

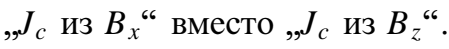

Возможная реализация такой методики приведена на рис. 10. МО пленка располагается перпендикулярно плоскости СП ленты, так, чтобы край пленки был на минимальном расстоянии от поверхности ленты. Плоскость МО пленки повернута на угол примерно $45^{\circ}$ относительно оси $z$. Это сделано для возможности получения зависимости $B_{x}(x)$, так как при угле $90^{\circ} B_{x}$ будет параллельна МО пленке, а при угле $0^{\circ}$ мы будем измерять $B_{x}$, что представляет слабый интерес. На том же рис. 10 приведена карта распределения магнитного поля в плоскости МО пленки.
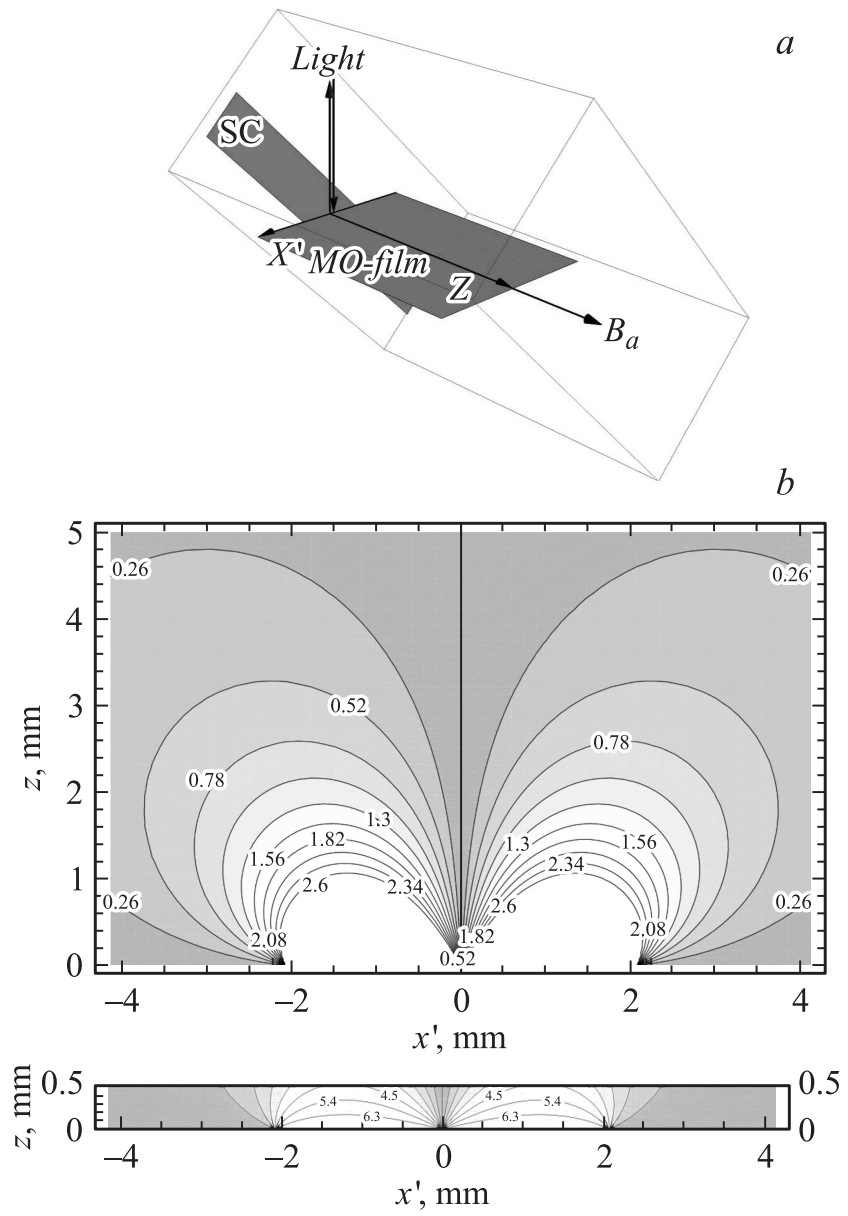

Рис. 10. Возможная реализация метода оценки „, $J_{c}$ из $B_{x}$ “: $a-$ геометрия эксперимента, $b-$ карты плотности для модуля касательной компоненты поля в плоскости $X^{\prime} Z$ в различных масштабах. 


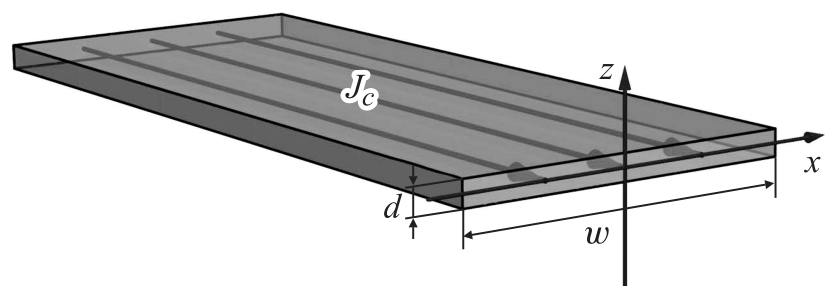

Рис. 11. Система координат, принятая при расчете.

Используя подход для оценки критического тока, описанный выше, из профилей магнитной индукции, полученных при различных температурах, можно найти температурную зависимость $J_{c}$.

Такие расчеты имеют не только фундаментальный, но и прикладной характер, так как позволяют корректно оценить величину плотность критического тока при пониженных температурах, где транспортные измерения сильно затруднены, в частности, из-за значительных абсолютных значений токов более $1 \mathrm{kA}$.

\section{4. Основные результаты и выводы}

Обобщим полученные результаты.

1. Предложен способ расчета распределения магнитного поля внутри и вовне сверхпроводящей ленты конечной толщины для различных участков кривой намагничивания в рамках модели $J_{c}=$ const.

2. Описан новый метод оценки критической плотности тока из наклона профиля поля в точке перегиба.

3. Асимметрия профиля поля, наблюдающаяся в эксперименте, объяснена непараллельностью МО пленки и сверхпроводящей ленты.

4. Предложено использовать метод оценки $J_{c}$ из измерений топографии $B_{x}$ компоненты поля, и приведена схема его возможной реализации.

Авторы выражают благодарность А.И. Подливаеву и А.В. Кузнецову за полезные обсуждения материалов работы.

М.А. Осипов и И.А. Руднев благодарят Российский научный фонд за поддержку исследований (проект № 1422-00098).

\section{Приложение A}

\section{Распределение магнитного поля в полоске с током}

Магнитное поле, создаваемое бесконечной полоской с поперечным сечением $d \times w$, несущей ток плотности $J$ (рис. 11) найдем из соотношения (2), записав его для системы координат, приведенной на рис. 11:

$$
\mathbf{B}_{\text {strip }}=\int_{-w / 2}^{+w / 2} \int_{-d / 2}^{+d / 2} \frac{\mu_{0}}{2 \pi} \frac{\left(0, J_{c}, 0\right)\left(x-x^{\prime}, 0, z-z^{\prime}\right)}{\left(x-x^{\prime}\right)^{2}+\left(z-z^{\prime}\right)^{2}} d x^{\prime} d z^{\prime} .
$$

Для компоненты $\left(B_{\text {strip }}\right)_{z} \equiv B_{z}$ после интегрирования получаем следующее выражение:

$$
\begin{aligned}
\tilde{B}_{z} & =\frac{B_{z}}{\mu_{0} J_{c} d / 2}=\frac{1}{2 \pi}-\frac{(\tilde{z}-1)}{2} \\
& \times \ln \left(\frac{(\tilde{z}-1)^{2}+a^{2}(\tilde{x}+1)^{2}}{(\tilde{z}-1)^{2}+a^{2}(\tilde{x}-1)^{2}}\right) \\
& -a(\tilde{x}+1) \operatorname{arctg}\left(\frac{\tilde{z}-1}{a(\tilde{x}+1)}\right) \\
& +a(\tilde{x}-1) \operatorname{arctg}\left(\frac{\tilde{z}-1}{a(\tilde{x}-1)}\right) \\
& +\frac{(1+\tilde{z})}{2} \ln \left(\frac{(\tilde{z}+1)^{2}+a^{2}(\tilde{x}+1)^{2}}{(\tilde{z}+1)^{2}+a^{2}(\tilde{x}-1)^{2}}\right) \\
& +a(\tilde{x}+1) \operatorname{arctg}\left(\frac{\tilde{z}+1}{a(\tilde{x}+1)}\right) \\
& -a(\tilde{x}-1) \operatorname{arctg}\left(\frac{\tilde{z}+1}{a(\tilde{x}-1)}\right) .
\end{aligned}
$$

Выражение для $B_{x}$ получается (в силу симметрии задачи) из формулы (ПА1) следующей циклической заменой:

$$
\begin{aligned}
& w \rightarrow d, d \rightarrow w, x \rightarrow z, z \rightarrow x, B_{z} \rightarrow-B_{x}, \\
\tilde{B}_{x} & =\frac{B_{x}}{\mu_{0} J_{c} d / 2}=\frac{1}{2 \pi}-\frac{(-\tilde{x}-1)}{2} \\
& \times \ln \left(\frac{(-\tilde{z}+1)^{2}+a^{2}(-\tilde{x}-1)^{2}}{(-\tilde{z}-1)^{2}+a^{2}(-\tilde{x}-1)^{2}}\right) \\
& -\frac{1}{a}(-\tilde{z}+1) \operatorname{arctg} a\left(\frac{-\tilde{x}-1}{-\tilde{z}+1}\right) \\
& +\frac{1}{a}(-\tilde{z}-1) \operatorname{arctg} a\left(\frac{-\tilde{x}-1}{-\tilde{z}-1}\right) \\
& +\frac{(-\tilde{x}+1)}{2} \ln \left(\frac{(-\tilde{z}+1)^{2}+a^{2}(-\tilde{x}+1)^{2}}{(-\tilde{z}-1)^{2}+a^{2}(-\tilde{x}+1)^{2}}\right) \\
& +\frac{1}{a}(-\tilde{z}+1) \operatorname{arctg} a\left(\frac{-\tilde{x}+1}{-\tilde{z}+1}\right) \\
& -\frac{1}{a}(-\tilde{z}-1) \operatorname{arctg} a\left(\frac{-\tilde{x}+1}{-\tilde{z}-1)}\right) .
\end{aligned}
$$

Здесь использованы следующие обозначения: $a=$ $=w / d-$ отношение ширины ленты к ее толщине (в рассматриваемом случае $\left.a \sim 10^{3} \gg 1\right) ; \tilde{x}=\frac{x}{(w / 2)}-$ нормированная координата по оси $X ; \tilde{z}=\frac{x}{(d / 2)}-$ нормированная координата по оси $Z$.

В случае если центр полоски расположен в точке $\left(x_{0}, z_{0}\right)$, в формулах (ПА1), (ПА2) необходимо сделать замену:

$$
x \rightarrow\left(x-x_{0}\right), \quad z \rightarrow\left(z-z_{0}\right) .
$$




\section{Приложение Б}

\section{„Собственное“ магнитное поле ленты В критическом состоянии (в „насыщении“)}

Магнитное поле создается критическими токами, текущими по всему сечению ленты. Подставляя в выражение для $B^{\prime}$ (см. формулу (5)):

$$
B^{\prime}(x, z)=B_{+}(x, z)+B_{-}(x, z)
$$

формулы из Приложения А, получим следующие соотношения:

$$
\begin{gathered}
B_{+}(x, z)=B_{\text {strip }}\left(\frac{x-w / 4}{w / 4}, \frac{z}{d / 2}, a / 2, J_{c}\right), \\
B_{-}(x, z)=B_{\text {strip }}\left(\frac{x+w / 4}{w / 4}, \frac{z}{d / 2}, a / 2,-J_{c}\right), \\
\tilde{B}_{+}(\tilde{x}, \tilde{z})=\frac{B_{+}}{\mu_{0} J_{c} d / 2}=\tilde{B}_{\text {strip }}(2 \tilde{x}-1, \tilde{z}, a / 2), \\
\tilde{B}_{-}(\tilde{x}, \tilde{z})=\frac{B_{-}}{\mu_{0} J_{c} d / 2}=-\tilde{B}_{\text {strip }}(2 \tilde{x}+1, \tilde{z}, a / 2) .
\end{gathered}
$$

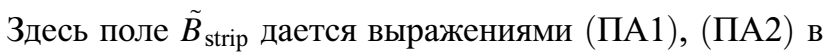
Приложении А, $\tilde{x}, \tilde{z}$ и $a$ соответствуют введенным там же обозначениям. Компоненты магнитного поля могут быть компактно записаны следующим образом:

$$
\begin{aligned}
\tilde{B}_{z}(\tilde{x}, \tilde{z})= & \frac{1}{2 \pi} \sum_{i, j=1}^{2}\left(f\left((-1)^{i} z-1, a\left((-1)^{j} x+1\right)\right)\right. \\
+ & \left.f\left((-1)^{i} z+1, a x\right)\right) \\
\tilde{B}_{x}(\tilde{x}, \tilde{z})= & \frac{1}{2 \pi} \sum_{i, j=1}^{2}\left(f \left(a \left((-1)^{i+j}\right.\right.\right. \\
& \left.\left.+(-1)^{i+1} \tilde{x}\right),(-1)^{i+1}+\tilde{z}\right) \\
& \left.+f\left(a(-1)^{i} \tilde{x},(-1)^{i+1}+\tilde{z}\right)\right) \\
f(p, q) & =\frac{p}{2} \ln \left(p^{2}+q^{2}\right)+q \operatorname{arctg}\left(\frac{p}{q}\right)
\end{aligned}
$$

\section{Приложение В}

\section{Лента на участке перемагничивания}

На участке перемагничивания распределение критических токов представлено на рис. 5, с. Собственное поле в образце равно сумме полей от четырех полос

$$
\begin{aligned}
B(x, z)= & -B_{\text {strip }}\left(\frac{\tilde{x}-1}{\tilde{l}_{p} / 2}+1, \tilde{z}, a \tilde{l}_{p} / 2\right) \\
& +B_{\text {strip }}\left(\frac{\tilde{x}+1}{\tilde{l}_{p} / 2}-1, \tilde{z}, a \tilde{l}_{p} / 2\right) \\
& +B_{\text {strip }}\left(\frac{\tilde{x}}{\left(1-\tilde{l}_{p}\right) / 2}-1, \tilde{z}, a\left(1-\tilde{l}_{p}\right) / 2\right) \\
& -B_{\text {strip }}\left(\frac{\tilde{x}}{\left(1-\tilde{l}_{p}\right) / 2}+1, \tilde{z}, a\left(1-\tilde{l}_{p}\right) / 2\right) .
\end{aligned}
$$

Здесь $\tilde{l}_{p}=l_{p}(w / 2)$.

Для расчета топографии поля необходимо найти положение $\tilde{l}_{p}$,фронта перемагничивания““.

Условие $B_{z}(0,0)=$ const приводит к следующему соотношению между внешним полем $B_{a}$, „собственным полем в центре образца $B_{z}^{\prime}(0,0)$, максимальным приложеным полем $B_{a, \max }$ и полем полного проникновения $B^{*}$

$$
B_{a}+B_{z}^{\prime}(0,0)=B_{a, \max }-B^{*}
$$

или

$$
B_{z}^{\prime}(0,0)=B_{a, \max }-B_{a}-B^{*} .
$$

Зная величину $B_{z}^{\prime}(, 0)$ можно определить положение фронта потока $\tilde{l}_{p}$ в сверхпроводнике

$$
\tilde{l}_{p}=\tilde{l}_{p}\left[B_{z}^{\prime}(0,0)\right]
$$

Более подробно можно записать

$$
\begin{aligned}
B_{z}^{\prime}\left(0,0, a, \tilde{l}_{p}\right) & =B_{a, \max }-B_{a}-B^{*} \\
& =B_{a, \max }-B_{a}-B_{z}^{\prime}(0,0, a, 0) .
\end{aligned}
$$

Разрешив уравнение (2) относительно $\tilde{l}_{p}$ и подставив его в формулу (1), можно получить распределение поля.

Поле в центре образца (в точке $(0,0))$ в зависимости от положения „фронта перемагничивания“ $\tilde{l}_{p}$ задается формулой

$$
\begin{aligned}
& \tilde{B}_{z}\left(0,0, a, \tilde{l}_{p}\right)=\frac{2}{\pi}\left(\frac{1}{2} \ln \left(a^{2}+1\right)-\ln \left(a^{2}\left(\tilde{l}_{p}-1\right)^{2}+1\right)\right. \\
& \left.+a\left(\operatorname{arcctg}(a)+2\left(\tilde{l}_{p}-1\right) \operatorname{arcctg}\left(a\left(1-\tilde{l}_{p}\right)\right)\right)\right) .
\end{aligned}
$$

Поле полного проникновения

$$
\tilde{B}^{*}=-\tilde{B}_{z}^{\prime}(0,0, a, 0)=\frac{2}{\pi}\left(\frac{1}{2} \ln \left(a^{2}+1\right)+a \operatorname{arctg}(a)\right) .
$$

При нахождении зависимости $\tilde{l}_{p}\left(\tilde{B}_{a}\right)$ использовалась следующая численная процедура. Для данного значения параметра а вычислялся набор точек $\left\{\tilde{B}_{z}\left(\tilde{l}_{p}\right), \tilde{l}_{p}\right\}$ и по нему строилась интерполяционная функция $\tilde{l}_{p}\left(\tilde{B}_{z}\right)$, описывающая зависимость положения фронта перемагничивания от поля в центре образца. Полученная функция $\tilde{l}_{p}\left(\tilde{B}_{z}\right)$ позволяет определить положение границ областей с однородным током и, следовательно, вычислить профиль поля. 


\section{Список литературы}

[1] Website of THEVA [Online]. Available: www.theva.com/

[2] Furtner S., Nemetschek R., Semerad R., Sigl G., Prusseit W. // Supercond. Sci. Technol. 2004. Vol. 17. P. S281-S284.

[3] Pokrovskiy S.V., Rudnev I.A., Podlivaev A.I., Kalitka V.S., Blednov A.V., Adamenkov A.A. // Scient. visualization. 2014. Vol. 6. N 5. P. 1-7.

[4] Fuger R., Hengstberger F., Eisterer M., Weber H.W. // IEEE Trans. Appl. Supercond. 2007. Vol. 17. N 2. P. 3753-3756.

[5] Higashikawa K., Shiohara K. // IEEE Trans. Appl. Supercond. 2012. Vol. 22. N 3. P. 5-8.

[6] Jooss C., Albrecht J., Kuhn H., Leonhardt S., Kronmuller H. // Rep. on Progr. Phys. 2002. Vol. 65. P. 651-788.

[7] Magneto-Optical Imaging / Ed. by T.H. Johansen, D.V. Shantsev. NATO Science Series II: Mathematics, Physics and Chemistry. 2014. Vol. 142.

[8] Villaume A., Porcar L., Bourgault D., Antonevici A., Caroff T., Leggeri J.P., Villard C. // Supercond. Sci. Technol. 2008. Vol. 21. P. 034009.

[9] Lucarelli F., Yang R., Lpke G., Haugan T.J., Levin G., Barnes P.N. // Supercond. Sci. Technol. 2008. Vol. 21. P. 115003.

[10] Song H., Davidson M.W., Schwartz J. // Supercond. Sci. Technol. 2009. Vol. 22. P. 062001.

[11] Caroff T., Porcar L., Chaudout P., Abrutis A., Jimnez C., Odier P., Weiss F. // IEEE Trans. Appl. Supercond. 2009. Vol. 19. P. 31843187.

[12] Jung Y., Kwak K., Lee W., Rhee J., Youm D., Yoo J., Han Y.H., Park B.J. // Supercond. Sci. Technol. 2012. Vol. 25. P. 065001.

[13] Machi T., Chikumoto N., Nakao K., Aoki Y., Kitoh Y., Fuji H., Izumi T., Ibi A., Yamada Y. // Physica C: Supercond. 2006. Vol. 445-448. P. 673676.

[14] Kamiya Y., Ishiyama A., Yagi M., Maruyama O., Ohkuma T. // IEEE Trans. Appl. Supercond. 2012. Vol. 22. P. 5801004.

[15] Abraimov D., Gurevich A., Polyanskii A., Cai X.Y., Xu A., Pamidi S., Larbalestier D., Thieme C.L.H. // Supercond. Sci. Technol. 2008. Vol. 21. P. 082004.

[16] Takao T., Koizuka S., Oi K., Ishiyama A., Kato J., Machi T., Nakao K., Shiohara Y. // IEEE Trans. Appl. Supercond. 2007. Vol. 17. P. 35173519.

[17] Villaume A., Porcar L., Bourgault D., Antonevici A, Caroff T., Leggeri J.P., Villard C. // Supercond. Sci. Technol. 2008. Vol. 21. P. 034009.

[18] Takao T., Koizuka S., Oi K., Ishiyama A., Kato J., Machi T., Nakao K., Shiohara Y. // IEEE Trans. Appl. Supercond. 2007. Vol. 17. P. 35173519.

[19] Руднев И.А., Осипов М.А. // Известия РАН. Сер. физическая. 2013. Т. 77. № 3. С. 369-372.

[20] Rudnev I., Osipov M. // J. Supercond. Novel Magn. 2014. Vol. 27. P. 951-954.

[21] Кашурников В.А., Руднев И.А., Зюбин М.В. // ЖЭТФ. 2002. T. 121. C. 442.

[22] Bean C.P. // Rev. Mod. Phys. 1964. Vol. 61. P. 31.

[23] Brandt E.H., Indenbom M.V., Forkl A. // Eurohp. Lett. 1993. Vol. 22. P. 735-740.

[24] Brandt E.H., Indenbom M.V. // Phys. Rev. B. 1993. Vol. 48. P. 12893-12906.

[25] Osipov M., Primenko A., Rudnev I. // IEEE Trans. Appl. Supercond. 2016. Vol. 26. N 3. P. 8001305. 